\title{
Philosophiques
}

\section{La théorie bolzanienne du fondement et de la conséquence}

\section{Armin Tatzel}

Volume 30, numéro 1, printemps 2003

Bernard Bolzano. Philosophie de la logique et théorie de la connaissance

URI : https://id.erudit.org/iderudit/007739ar

DOI : https://doi.org/10.7202/007739ar

Aller au sommaire du numéro

Éditeur(s)

Société de philosophie du Québec

ISSN

0316-2923 (imprimé)

1492-1391 (numérique)

Découvrir la revue

Citer cet article

Tatzel, A. (2003). La théorie bolzanienne du fondement et de la conséquence. Philosophiques, 30(1), 191-217. https://doi.org/10.7202/007739ar

\section{Résumé de l'article}

Le but de cet article est de présenter et d'évaluer la théorie de la fondation de Bernard Bolzano, c'est-à-dire sa théorie du concept exprimé et de la relation mise en jeu par " parce que ». Dans la première partie (\$§1-4), le concept de fondation est distingué et mis en relation avec trois autres concepts : le concept de raison épistémique, le concept de causalité et le concept de déductibilité (c'est-à-dire de conséquence logique). Dans la seconde partie (§§5-7), je reconstruis la théorie bolzanienne de la fondation sous forme axiomatique et j'en offre une discussion critique.
Ce document est protégé par la loi sur le droit d'auteur. L'utilisation des services d’Érudit (y compris la reproduction) est assujettie à sa politique d'utilisation que vous pouvez consulter en ligne.

https://apropos.erudit.org/fr/usagers/politique-dutilisation/ 


\title{
La théorie bolzanienne du fondement et de la conséquence'
}

\author{
ARMIN TATZEL \\ Université de Genève \\ armin.tatzel@lettres.unige.ch
}

\begin{abstract}
RÉSUMÉ. - Le but de cet article est de présenter et d'évaluer la théorie de la fondation de Bernard Bolzano, c'est-à-dire sa théorie du concept exprimé et de la relation mise en jeu par «parce que». Dans la première partie ( $\left.\iint 1-4\right)$, le concept de fondation est distingué et mis en relation avec trois autres concepts : le concept de raison épistémique, le concept de causalité et le concept de déduc-

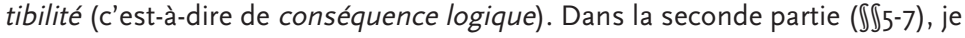
reconstruis la théorie bolzanienne de la fondation sous forme axiomatique et j'en offre une discussion critique.
\end{abstract}

\begin{abstract}
The aim of the paper is to present and evaluate Bernard Bolzano's theory of grounding, i.e. his theory of the concept expressed and the relation

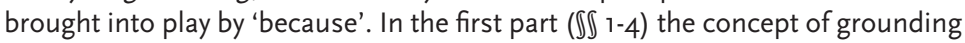
is distinguished from and related to three other concepts : the concept of an epistemic reason, the concept of causality and the concept of deducibility (i.e. logical consequence). In its second part ( $\iiint_{5-7)}$ Bolzano's positive account of grounding is reconstructed in axiomatic form and critically discussed.
\end{abstract}

\section{Fondation}

Il est notoire que la théorie de la déductibilité (Ableitbarkeit) de Bernard Bolzano - qui est en réalité une théorie de ce que l'on appelle aujourd'hui la «conséquence logique» - a anticipé à maints égards les travaux de Tarski sur le sujet ${ }^{2}$. Il est cependant beaucoup moins connu que Bolzano développe également une théorie de la relation de raison à conséquence ou de fondation (Abfolge), c'est-à-dire une théorie du concept exprimé et de la relation que met en jeu le connecteur "parce que» (weil). Prises ensemble, la déductibilité et la fondation constituent le cœur de sa théorie logique : Bolzano affirme que pour être en mesure de le comprendre, il faut être familier avec ces deux concepts $^{3}$. Dans cet article, je me pencherai sur le second ${ }^{4}$.

1. Une version anglaise de cet article paraîtra dans Notre Dame Journal of Formal Logic sous le titre : «Bolzano's Theory of Ground and Consequence».

2. Pour une discussion détaillée de la notion bolzanienne de déductibilité, voir l'article de Mark Siebel dans le même numéro. Note de l'éditeure.

3. Cf. Bolzano, 1851, p. 40.

4. Le lien étroit entre la conception de la fondation de Bolzano et la théorie des preuves normales de Gentzen (cf. Gentzen, 1969) est exploré plus en détails dans Tatzel (à paraître). 
La théorie de la fondation de Bolzano prend la forme d'une théorie sémantique des énoncés de la forme [p parce que q]. Elle s'appuie sur l'hypothèse de base selon laquelle, pris littéralement, les énoncés de la forme

$\lceil\mathrm{p}$ parce que $\mathrm{q}\rceil$

sont vrais seulement si

FONDE (P, Q)

où «FONDE» tient lieu d'une certaine relation —la relation de fondationet où « $\mathrm{P}$ » et « $\mathrm{Q}$ » désignent des termes singuliers qui dénotent les propositions exprimées par $« \mathrm{p} »$ et $« \mathrm{q}{ }^{5}$. Bolzano présente donc la fondation comme consistant en une relation entre des propositions ou, plus précisément, entre des propositions vraies. La fondation, selon lui, est une relation entre des vérités (Wabrheiten an sich). J'assumerai, tout au long de l'article, que le lecteur est familier avec les postulats fondamentaux de la sémantique bolzanienne ${ }^{6}$.

Je suivrai également Bolzano en considérant les propositions - ses célèbres Sätze an sich-comme des entités indépendantes de l'esprit et du langage qui sont les porteurs primitifs des propriétés vrai et faux, qui n'ont pas de pouvoir causal, et qui figurent à la fois comme contenu de pensée et comme significations des énoncés ${ }^{7}$. À l'instar de Bolzano, je considère que les vérités ne sont rien d'autre que des propositions vraies.

Bolzano désigne respectivement par «fondement (objectif) » (fondement $=$ Grund) et «conséquence (objective) » (conséquence = Folge) les termes de la relation de fondation. (Il $n$ 'utilise pas «conséquence» en relation avec la déductibilité, c'est-à-dire sa conception spécifique de... la conséquence $\operatorname{logique}^{8}$ !) Sa théorie de la fondation peut par conséquent également être appelée une théorie du fondement et de la conséquence. Il donne comme exemple ${ }^{9}$ :

(1) Les thermomètres qui fonctionnent bien montent plus haut l'été que l'hiver parce qu'il fait plus chaud l'été que l'hiver

5. Cf. Bolzano, 1837, $\mathbb{S}$ 168, 177. De manière générale, j’utilise «P», «Q $Q$ », .., comme désignateurs pour les termes dénotant des propositions et «p», "q»... comme désignateurs pour les énoncés déclaratifs exprimant ces propositions.

6. Notons que Bolzano ne dit pas que "parce que " est un prédicat dyadique connectant deux termes singuliers plutôt qu'un connecteur propositionnel. Pour cette raison il est à proprement parler faux de dire que «parce que » dénote, selon Bolzano, une relation. Néanmoins, Bolzano est forcé d'assumer que les énoncés de la forme [FONDE (P, Q) ] ont les mêmes conditions de vérité que les énoncés de la forme $\lceil\mathrm{p}$ parce que q]. De plus, il semble aussi faire l'hypothèse forte que les énoncés de la forme [FONDE (P, Q) † (ou ceux d'une forme semblable) révèlent la signification des énoncés de la forme 「p parce que q] (Bolzano, 1837, $\mathbb{\$} 177$, voir également $\$ 127)$. Nous pouvons donc dire que, même si Bolzano est conscient du fait que «parce que " est un connecteur qui ne dénote rien, ce dernier correspond de près au prédicat (artificiel) «FONDE» (que nous allons élucider dans la suite de l'article) et qui, lui, dénote une relation. Pour résumer cela, je dirai que «parce que» met en jeu la relation de fondation.

7. Pour la conception des propositions de Bolzano, Bolzano, 1837, $\mathbb{S} \mathbb{S} 19$ s., Textor, 1996, p. 9-28, et Künne, 1997. Pour sa conception de la vérité, Bolzano, 1837, $\mathbb{S} 25 \mathrm{~s}$.

8. Nous reviendrons sur la déductibilité et sa relation à la fondation à la section 4 .

9. Cf. Bolzano, 1837, $\mathbb{S} 162$. 
qui est manifestement vraie, tandis que sa converse

(2) Il fait plus chaud l'été que l'hiver parce que les thermomètres qui fonctionnent bien montent plus haut l'été que l'hiver

est manifestement fausse. (Pour simplifier, on a ignoré ici les considérations reliées à la pression atmosphérique.) En utilisant les crochets carrés pour dénoter les propositions exprimées par les énoncés (entre guillemets), nous pouvons dire, conformément à l'hypothèse sémantique de base de Bolzano, que (1) dit que la vérité [Il fait plus chaud l'été que l'hiver] est le fondement (partiel) de la vérité [Les thermomètres qui fonctionnent bien montent plus haut l'été que l'hiver], qui est elle-même la conséquence (partielle) de la première; et cela semble être vrai. Puisque (2) est fausse, la converse ne fonctionne pas : la seconde vérité n'est pas le fondement (partiel) de la première, et la première n'est pas la conséquence (partielle) de la seconde.

Si l'hypothèse de Bolzano à propos du caractère relationnel des énon-

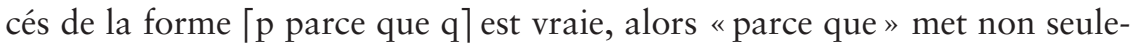
ment en jeu la relation de fondation, mais il le fait en exprimant un certain concept, en l'occurrence [parce que ${ }^{10}$. (Je prends pour acquis - suivant Bolzano - que les concepts sont les constituants des propositions; je généralise ici l'usage des crochets carrés de manière évidente.) La théorie de Bolzano porte tant sur le concept [parce que] que sur la relation de fondation elle-même. Bien que cela eût parfois été pertinent, Bolzano n'est pas toujours explicite quant au tracé de cette distinction, mais il en demeure néanmoins conscient ${ }^{11}$. Les passages concernés ne laissent en général aucune doute sur la question de savoir s'il met l'emphase sur le concept ou sur la relation.

Les investigations de Bolzano sur le concept exprimé et la relation que met en jeu "parce que» sont principalement contenues dans la Wissenschaftslehre. Voici un aperçu de la théorie du fondement et de la conséquence qui s'y trouve; (les chiffres, à droite, indiquent les sections correspondantes dans le présent article) :

I. Explications et comparaisons informelles

La représentation générale $(\mathbb{S} 162,168,198)(1$.

Fondements objectifs versus raisons épistémiques $(\mathbb{S} 177,198)(2$.

Fondation versus causalité $(\mathbb{S} 168.2,201)(3$.

Fondation versus déductibilité $(\mathbb{S} 162,200)$ (4.)

II. Fondation immédiate $(\mathbb{S}$ 203-213) (6.)

III. Fondation médiate $(\mathbb{S}$ 214-219) (7.)

IV. Critères pour la fondation $(\mathbb{2} 21)$

10. Bolzano utilise parfois malencontreusement le verbe «dénoter» (bezeichnen) pour la relation entre l'expression et le concept qu'elle exprime (Bolzano, 1837, $\$ 285$ ).

11. Cf.Bolzano, 1837, $\$ 162$ où Bolzano introduit la relation de fondation, et Bolzano, $1837, \mathbb{} 198$ où il introduit le concept exprimé par "parce que". 
Dans les passages subsumés en I, Bolzano tente de mettre en lumière l'idée de fondation en la comparant avec trois autres idées apparentées et en faisant ressortir les liens entre elles. J'examine cette comparaison dans les sections 2. à 5. Dans les sections 6. et 7. je me penche sur la conception spécifiquement bolzanienne de la fondation (II et III) que je formule en faisant appel aux définitions implicites de deux concepts apparentés : la fondation immédiate et la fondation médiate. Les idées de Bolzano quant aux conditions sous lesquelles la relation de fondation est réalisée (IV) sont discutées ailleurs ${ }^{12}$.

\section{Fondement objectif versus raisons épistémiques}

Bolzano utilise le mot "raison épistémique " (Erkenntnisgrund) en deux sens (connexes). Je les nommerai sens «psychologique» et sens «propositionnel». Une collection ${ }^{13}$ de jugements (Urteile) x est la raison épistémique au sens psychologique d'un certain jugement y si et seulement si y est vrai et $\mathrm{x}$ est la cause complète de $\mathrm{y}$ (et $\mathrm{x}$ et $\mathrm{y}$ se produisent dans l'esprit d'une seule et même personne $)^{14}$. Dans cette définition, «jugement» doit bien entendu être pris comme dénotant des événements mentaux particuliers qui se produisent dans l'esprit d'une certaine personne à un certain moment. Le jugement d'un astronome d'une époque antérieure à l'effet que la terre est ronde peut avoir été causé (en partie) par son jugement que l'ombre de la terre sur la lune est ronde. Le cas échéant, le second jugement aurait été la raison épistémique (partielle) du premier. On doit toutefois noter une chose en ce qui concerne cette définition : par «la collection des jugements x est la cause complète du jugement y ", Bolzano veut simplement dire qu'il n'y a pas d'autres jugements qui soient causalement responsables de l'occurrence de y. Il ne veut pas dire qu'il n'y a rien d'autre qui soit responsable de l'occurrence de $\mathrm{y}^{15}$.

Les fondements objectifs sont clairement distincts des raisons épistémiques au sens psychologique. Les raisons épistémiques au sens psychologique sont des (collections de) jugements, et ont donc des pouvoirs causaux. Les fondements objectifs sont des (collections de) propositions et n'ont donc pas de pouvoirs causaux ${ }^{16}$.

Les raisons épistémiques au sens propositionnel sont les contenus des raisons épistémiques au sens psychologique. Plus précisément, une collection de propositions $\alpha$ est la raison épistémique au sens propositionnel de la proposi-

12. Cf. Tatzel, à paraître.

13. Une collection (Inbegriff, Bolzano, 1837, $\mathbb{8} 82$ ) est, en gros, un tout constitué de parties. J'en dirai un peu plus long sur les collections dans la section 5.

14. Bolzano introduit le terme «Erkenntnisgrund» dans ce sens dans Bolzano, 1837, \$313. J'ai ajouté la partie de la définition entre crochets à la formulation officielle de Bolzano. Bien qu'il ne mentionne pas cette condition explicitement, il est évident qu'il la présuppose.

15. Cf. Bolzano, 1837, $\mathbb{\$} 300.2$.

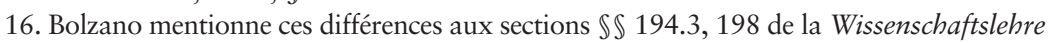
et dans Bolzano, Gesamtausgabe vol. 2A7 p. 81. 
tion $\beta$ si et seulement si il y a une collection de jugements (particuliers) $\mathrm{x}$ et un unique jugement (particulier) y tel que les propositions qui font partie de $\alpha$ sont les contenus des jugements qui font partie de $\mathrm{x}$ (et tel que les parties de $\alpha$ et les parties de $\mathrm{x}$ sont en relation de bijection), $\beta$ est le contenu de $\mathrm{y}$, et $\mathrm{x}$ est la raison épistémique (psychologique) de $\mathrm{y}^{17}$. Ainsi, s'il y a déjà eu un astronome qui a raisonné tel que je l'ai décrit plus haut, la proposition que l'ombre de la terre sur la lune est ronde est la raison épistémique (partielle) de la proposition que la terre est ronde.

La distinction catégorielle mentionnée plus haut entre les fondements objectifs et les raisons épistémiques au sens psychologique ne s'applique pas entre les fondements objectifs et les raisons épistémiques au sens propositionnel puisqu'ils sont tous deux des (collections de) propositions. Mais la propriété d'être une raison épistémique au sens propositionnel est néanmoins différente de la propriété d'être un fondement objectif, et, mutatis mutandis, la relation de fondation est différente de la relation qu'il y a entre une certaine raison épistémique et la proposition dont elle est la raison épistémique, et ce pour plusieurs raisons (dans le reste de cette section, j'utilise le mot «raison épistémique » au sens propositionnel) :

1. La proposition que la terre est ronde peut être la raison épistémique (partielle) de la proposition que l'ombre de la terre sur la lune est ronde et vice versa. Cependant, malgré que la proposition précédente soit le (ou une partie du) fondement objectif de la seconde, l'inverse n'est pas le cas ${ }^{18}$. On peut affirmer la même chose, par exemple, en ce qui concerne l'exemple du thermomètre mentionné dans la première section.

2. Une vérité donnée peut avoir une multitude de raisons épistémiques différentes. Différentes personnes à différents moments peuvent la reconnaître de différentes manières. En revanche, Bolzano prétend qu'il est impossible qu'une vérité (ou une collection de vérités) repose sur des fondements objectifs (entièrement) différents. Cela, ajoute Bolzano, n'empêche pas le cas où une vérité repose sur différentes (collections de) propositions qui figurent comme parties de son unique fondement objectif ${ }^{19}$. Il soutient donc que si une vérité donnée semble reposer sur des fondements objectifs différents, ces derniers devraient toujours être traités comme différentes parties d'un fondement objectif unique et complet. (Bolzano présuppose ici un concept de fondation très strict et très restreint. On pourra douter que notre usage ordinaire de «parce que » est lié à $c e$ concept. Voir mes remarques à la fin de la section 3.)

17. Bolzano utilise le terme "Erkenntnisgrund» au sens propositionnel aux sections $\mathbb{S} \mathbb{S} 177,194.3,198$ de la Wissenschaftslehre, de même que dans Bolzano, 1841, p. 68, et Bolzano, 1834, vol. I, p. 6.

18. Cf. Bolzano, 1837, $\$ 209$.

19. Cf. Bolzano, 1837, $\$ 206$; Bolzano, 1841, p. 68. Je ferai état de mes réserves à cet égard à la section 6 . 
3. Il est possible de connaître une certaine vérité via (le jugement d') une raison épistémique quelconque sans connaître son fondement objectif. Quelqu'un peut savoir que $E=m c^{2}$, ou même qu'il fait plus chaud aujourd'hui qu'hier pour une raison épistémique quelconque (par le concours d'un témoignage, par exemple) sans savoir quoi que ce soit à propos du fondement objectif sur lequel repose la vérité en question ${ }^{20}$. Il arrive fréquemment que des scientifiques savent d'une collection de propositions qu'elles sont vraies mais non pourquoi elles le sont, et c'est d'ailleurs le plus souvent ce qu'ils cherchent à savoir.

4. Les fondements (et conséquences) objectifs doivent être des (collections de) propositions vraies, tandis que les raisons épistémiques peuvent être fausses ou avoir des parties qui sont des propositions fausses.

5. Il est possible (et c'est aussi probablement le cas) qu'il y ait des fondements objectifs qui n'ont jamais été et ne seront jamais le contenu d'aucun jugement particulier. Mais il n'est pas possible qu'il y ait des raisons épistémiques qui n'aient jamais été et qui ne seront jamais le contenu d'aucun jugement particulier.

Bolzano mentionne uniquement les trois premières raisons de façon explicite. Mais il les accepterait assurément toutes.

Nous utilisons parfois un énoncé de la forme 「p parce que q] pour dire que $\mathrm{Q}$ est une raison épistémique de P. Mais ces usages, soutient Bolzano, ne sont pas des usages corrects. Que pourrait bien vouloir dire quelqu'un qui, en plein milieu d'une réflexion, énonce avec sincérité un énoncé telle que "Il fait plus chaud l'été que l'hiver parce que les thermomètres qui fonctionnent bien montent plus haut l'été que l'hiver»? «En disant cela, nous ne parlons que de la cognition de ces vérités, [...] nous voulons simplement indiquer que la cognition de l'une de ces vérités cause la cognition de l'autre » ${ }^{21}$. À cette étape de l'enquête, nous devons nous en tenir à cet argument de plausibilité, mais dans la section suivante, nous verrons qu'il y a davantage à dire en faveur de l'affirmation de Bolzano.

20. Cf. Bolzano, 1841, p. 77; Bolzano, 1834, vol. I, p. 6.

21. Bolzano, 1837, $\mathbb{1 9 8}$; Malheureusement, un nombre dérisoire de textes bolzaniens — pas même la Wissenschaftslehre! —ont été traduits en français jusqu'à maintenant. Les traductions de l'allemand sont de Sandra Lapointe. Je traduis «Inbegriff» par «collection» (et non par «classe»). Mes motivations apparaîtront à la section 5. De plus, je traduis "Erklärung" par «analyse» (et non par "définition») parce que l'intention de Bolzano lorsqu'il propose ce qu'il appelle une «Erklärung» est d'expliquer les concepts (plus ou moins) ordinaires que nous possédons et utilisons déjà, et non d'en stipuler de "nouveaux". Bolzano a une conception méréologique des concepts selon laquelle les concepts ont des parties. Ces parties peuvent être d'autres concepts ou encore des intuitions. (Cf. Bolzano, 1837, $\mathbb{S}$ 72, 73). L'analyse est, dans cette perspective, un type d'explication particulier : analyser un concept consiste à en donner une explication en identifiant ses parties et la manière dont elles sont combinées. Pour le concept bolzanien d'analyse, Bolzano, 1837, $\$ 554$. 


\section{Fondation versus causalité}

Un autre « concept très apparenté à celui de fondation (Abfolge) [c'est-à-dire, le concept exprimé par "parce que »] est sans aucun doute celui de causalité, c'est-à-dire celui de la relation entre les causes et les effets. Aussi, l'un de ces concepts pourrait-il peut-être être utilisé pour définir l'autre? " ${ }^{22}$. Demandonsnous dans un premier temps si [parce que] peut être défini (en partie) via le concept de causalité.

Nous pourrions répondre franchement par l'affirmative si [parce que] était - comme le dit Bolzano - subordonné au concept de causalité. (Dans la terminologie bolzanienne, un concept A est subordonné au concept B (A ist $\mathrm{B}$ untergeordnet) si et seulement si tout ce qui tombe sous $\mathrm{A}$ tombe aussi sous B, mais pas l'inverse ${ }^{23}$. Or, cela ne peut manifestement pas être le cas. Les fondements et conséquences ne peuvent être les causes et effets puisqu'ils sont des vérités et n'ont par conséquent aucun pouvoir causal ${ }^{24}$.

Mais même si [parce que] n'est pas subordonné à [cause], le premier pourrait tout de même être défini par le second. Intuitivement, il semble plausible de concevoir la connexion entre les deux concepts de la façon suivante : «les vérités qui affirment l'existence et les propriétés (Beschaffenheiten) d'une cause doivent être considérées comme le fondement, et celles qui concernent l'existence et les propriétés de l'effet comme la conséquence » ${ }^{25}$. On aurait alors affaire à un modèle à deux niveaux : d'une part, le niveau des choses concrètes "dans le monde" qui sont reliées causalement les unes aux autres. D'autre part, le niveau propositionnel abstrait dans lequel les connexions causales sont "reflétées" par les propositions vraies à propos des choses "dans le monde» et des relations causales qu'il y a entre elles : les vérités reflétant les connexions causales entre les choses sont elles-mêmes reliées comme fondements et conséquences objectifs.

Bolzano ne rejette pas le modèle à deux niveaux. Il insiste toutefois sur un point particulier qui, malgré qu'il ait été négligé par les partisans du modèle à deux niveaux, semble être d'un grand intérêt : il y a des paires de fondements et conséquences objectives qui ne reflètent pas des relations d'ordre causal.

Les vérités mathématiques ne portent pas sur des objets réels. Mais ne doiventelles pas être reliées comme fondement et conséquence? La vérité que dans un triangle équilatéral tous les angles sont égaux de doit-elle pas être une conséquence de la vérité qu'un triangle isocèle possède deux angles égaux ${ }^{26}$ ?

L'affirmation de Bolzano ne semble pas valoir uniquement que pour les vérités mathématiques. Il semble en effet que nous puissions poser des questions de la forme "pourquoi...? " à l'égard de tous les types de propositions

22. Bolzano, 1837, $\mathbb{} 201$.

23. Cf. Ibid., $\mathbb{} 97$.

24. Cf. Ibid., $\mathbb{S} 201.2$.

25. Ibid., $\$ 201.3$.

26. Ibid., $\mathbb{} 201.3$. 
qui ne contiennent aucun effet et auxquelles on peut répondre en formulant des énoncés de la forme [q parce que p $\rceil$ au sens propre. Voici quelques candidats plausibles :

- $\quad$ Soit Bush, soit Gore a gagné les élections parce que Bush les a gagnées.

- Ce verre contient des molécules d'eau parce qu'il contient des molécules de $\mathrm{H}_{2} \mathrm{O}$.

- Pierre est le cousin de Paul parce qu'il est le fils d'un frère ou d'une sœur, soit de la mère, soit du père de ce dernier.

- Le paradoxe de Russell est dérivable parce que le cinquième axiome de Frege est faux.

- $\quad$ Un énoncé de la forme [P et Q] est vrai (s’il est vrai) parce que les énoncés $\mathrm{P}$ et $\mathrm{Q}$ sont tous deux vrais.

- Un énoncé de la forme [Il est vrai que p广 est vrai (s'il est vrai) parce que l'énoncé $\mathrm{P}$ correspondant est vrai.

- $\quad$ Cette pomme est rouge parce que sa peau est rouge.

- $\quad$ Il devrait être puni parce qu'il a commis un crime.

- Il n’y a pas de carrés ronds parce que le concept d'un carré rond implique une contradiction.

- $\quad$ Socrate\} existe parce que Socrate existe ${ }^{27}$.

- $\quad$ Socrate exemplifie la pâleur parce qu'il est pâle.

On doit concéder que Bolzano n'aurait pas accepté les deux derniers exemples. Il aurait rejeté l'avant-dernier parce qu'il ne dispose pas du concept d'ensemble, et le dernier exemple aurait été rejeté parce que, si on le suit, les deux énoncés reliés par "parce que » expriment ici une seule et même proposition $^{28}$.

Nous avons débuté cette section par l'hypothèse selon laquelle [parce que] pourrait être subordonné à [cause]. Nous ne sommes pas parvenus à infirmer cette hypothèse, mais nous sommes parvenus à un résultat semblable. Même si [cause] n'est pas subordonné à [parce que] (puisque les objets qui tombent sous ces concepts appartiennent à différents "niveaux»), la relation de fondation subsiste également entre les propositions qui ne reflètent pas des causes et effets. Contrairement à ce que nous avions d'abord suggéré, on pourrait donc suspecter que, à l'inverse, [cause] doive bien plutôt être défini (en partie) sur la base de [parce que]. Et c'est aussi ce que Bolzano assume : «Nous devons dériver nos concepts de cause et d'effet de ceux que fondement

27. Fabrice Correia (à paraître) propose une explication de la relation qu'il y a entre Socrate et $\{$ Socrate\} lorsque la proposition exprimée par cet énoncé est vraie. Cette relation de "fondation ontologique " est, bien entendu, une proche parente de la relation de fondation «propositionnelle» qui nous occupe ici. À mon avis, la conception de la fondation ontologique de Correia sied en fait très bien la conception de la fondation propositionnelle de Bolzano.

28. Cf.Bolzano, 1837, $\mathbb{} 127$. 
et conséquence ${ }^{29}$. Sa suggestion quant à la manière dont cela doit être effectué est la suivante :

«X est la cause de $\mathrm{Y}$ » veut en fait dire; «la vérité que $\mathrm{X}$ existe $[$ daß X sey] est reliée à la vérité que $\mathrm{Y}$ existe comme le fondement (ou fondement partiel) à sa conséquence (ou conséquence partielle) ${ }^{30}$.

On pourrait croire que cela ne peut être vrai pour la raison suivante : il semble être vrai que \{Socrate\} existe parce que Socrate existe, mais Socrate n'est sûrement pas la cause de \{Socrate\}. Mais cette objection échoue : elle repose sur une mauvaise compréhension de ce que Bolzano dit réellement dans le passage cité. Pour bien le comprendre, il faut voir que Bolzano utilise «existence» (Dasein) comme synonyme de «réalité » (Wirklichkeit), par quoi il signifie la capacité d'agir sur quelque chose (Wirksamkeit) ${ }^{31}$. Ainsi la citation devrait être paraphrasée ainsi :

[X est la cause de $\mathrm{Y}]=$ [la vérité $[\mathrm{X}$ a la capacité d'agir sur quelque chose] est le fondement (partiel) de la vérité [Y a la capacité d'agir sur quelque chose] qui est sa conséquence (partielle)].

Selon cette interprétation, il est manifeste que si $\mathrm{X}=$ Socrate et $\mathrm{Y}=\{$ Socrates $\}$, la deuxième partie du contre-exemple ne peut désigner une vérité. L'objection peut alors être rejetée.

Mettons maintenant en relation les résultats des deux dernières sections. Qu'exprime "parce que » dans son usage littéral ? Est-ce que cela exprime : (1) le concept de fondation, (2) le concept de causalité ou encore (3) le concept de raison épistémique? Si la théorie causale de l'esprit doit être exacte, alors (3) doit être expliquée (en partie) sur la base de (2). Si, de plus, la thèse de Bolzano (ou une autre semblable) sur les liens entre fondements (et conséquences) et causes (et effets) est vraie, alors (2) doit, à son tour, être expliquée (en partie) sur la base de (1). Étant donné ces hypothèses, le concept de fondation ressort comme «le plus fondamental» des trois. Ainsi, si "parce que » n'a qu'une seule signification littérale, le concept de fondation semble être le premier choix pour remplir cette fonction.

Après avoir introduit les termes de "fondement " et "conséquence " sur la base d'exemples intuitifs tels que celui du thermomètre, Bolzano se restreint à un usage très précis et technique de ces termes : il utilise la plupart du temps "fondement» et "conséquence» pour les fondements et conséquences immédiats et complets. (Rappelons-nous les doutes exprimés concernant le second argument de Bolzano au sujet de la différence entre fondements objectifs et raisons épistémiques, argument dans lequel il fait un usage explicite de son concept strict de fondement en présupposant que des (collections

29. Cf. Ibid., $\mathbb{} 201.4$

30. Ibid., $\mathbb{S} 168$; voir aussi Bolzano, Gesamtausgabe vol. 1 16/2, p. 238.

31. Cf. Bolzano, 1838, p. 85 - qui montre également que Bolzano considère cette façon de parler comme étant justifiée par l'usage du langage ordinaire. 
de) vérités ne peuvent avoir plus qu'un seul fondement.) Je suis en désaccord avec Bolzano. Je crois qu'un concept moins strict qui embrasse également les fondements médiats et partiels convient beaucoup mieux à notre usage ordinaire de "parce que». Je fournirai des arguments à cet effet à la fin de cet article.

J'apporte ces précisions pour faire ressortir le point suivant : même si on a raison de croire que "parce que ", dans son usage littéral, exprime un certain concept (non-épistémique et non-causal) de fondation, il n'est pas clair quel est exactement celui qu'il exprime. Il y a plusieurs manières possibles de fixer le concept qui correspond, de prime abord, aux intuitions de Bolzano. On peut donc penser que "parce que » est univoque, et qu'il exprime un concept (non-épistémique, non-causal) de fondation, sans toutefois penser qu'il exprime le concept trop strict de fondation complète et immédiate.

\section{Fondation versus déductibilité}

Dans la célèbre section $\mathbb{S} 155$ de la Wissenschaftslehre, Bolzano donne la définition suivante ${ }^{32}$ :

Je dis que les propositions $\mathrm{M}, \mathrm{N}, \mathrm{O}, \ldots$ sont déductibles des propositions $\mathrm{A}, \mathrm{B}$, $\mathrm{C}, \mathrm{D}, \ldots$ relativement aux parties variables $\mathrm{i}, \mathrm{j}, \ldots$, si [et seulement si] chaque collection de représentations dont la substitution pour $\mathrm{i}, \mathrm{j}, \ldots$ fait en sorte que $\mathrm{A}$, $\mathrm{B}, \mathrm{C}, \mathrm{D}$, sont toutes vraies, rend aussi $\mathrm{M}, \mathrm{N}, \mathrm{O}, \ldots$ vraies $^{33}$.

J'expliquerai cela en utilisant un argument encore plus notoire :

Tous les hommes sont mortels.

Socrate est un homme.

Donc, Socrate est mortel.

La proposition exprimée par la conclusion de cet argument est déductible des propositions exprimées par ses prémisses, par exemple, à l'égard de la représentation [homme]. Prenons la variation $\mathrm{i} \% / \mathrm{i}$ de la proposition $\mathrm{P}$, à savoir la proposition $\mathrm{P}^{*}$ qui est le résultat de la substitution de la représentation i* pour (toutes les "occurrences» de) la représentation i en P. Pour chaque représentation $\mathrm{i}^{*}$, si les variantes $\mathrm{i} /[$ [homme] de [Tous les hommes sont mortels] et de [Socrate est un homme] sont vraies, alors la variante i*/[homme] de [Socrate est mortel] est vraie également. Cela signifie que

32. L'ouvrage le plus précis et le plus complet sur le concept de déductibilité est celui de Mark Siebel (1996). (On consultera aussi l'article sur le sujet publié dans ce numéro par le même auteur. Note de l'éditeure.)

33. Bolzano, 1837, $\$ 155.2$; L'ajout de « [et seulement si] » est justifié par le contexte du passage cité. Cf. Siebel, 1996, p. 14. Les représentations (Vorstellungen an sich) sont ces parties des propositions qui ne sont pas elles-mêmes des propositions. Cf. Bolzano, 1837, $\mathbb{\$} 48$. Bolzano examine la connexion entre la relation de fondation et la déductibilité principalement aux sections $\mathbb{S} \mathbb{S} 162$ et 200 de la Wissenschaftslehre. Dans les sections $\mathbb{S}$ 203-221, Bolzano caractérise la relation de fondation en la comparant à la déductibilité. Buhl (1961, p. 25-28) traite aussi du lien entre fondation et déductibilité. 
[Socrate est mortel] est déductible de [Tous les hommes sont mortels] et [Socrate est un homme] à l'égard de la représentation [homme]. Et cela vaut également pour les représentations [mortel] et [Socrate]. Ainsi, la proposition exprimée par la conclusion de cet argument est déductible des propositions exprimées par ses prémisses à l'égard de ces trois représentations.

Dans le passage cité plus haut, Bolzano définit le prédicat triadique fondamental « $\beta$ est déductible de $\alpha$ relativement à $i$ ». Mais on peut aisément en dériver un prédicat dyadique qui nous sera utile pour les fins de la présente discussion : la collection de propositions $\beta$ est déductible de la collection propositions $\alpha$ si et seulement si il y a au moins une représentation i telle que $\beta$ est déductible de $\alpha$ à l'égard de i. Bolzano lui-même utilise ce prédicat dyadique dérivé lorsqu'il compare la relation de fondation avec la relation de déductibilité. Je le suivrai donc en cela ${ }^{34}$ et par "relation de déductibilité », je réfèrerai tout au long de cette section à la relation dyadique.

On pourrait vouloir suggérer que la relation de fondation n'est rien d'autre que la relation de déductibilité. Mais cela ne saurait être possible pour une raison évidente : la relation de fondation ne subsiste qu'entre des vérités tandis que la relation de déductibilité peut avoir également des propositions fausses comme termes ${ }^{35}$. Pour cette raison, la comparaison la plus intéressante semble être celle entre la relation de fondation et la T-déductibilité, qui se définit comme suit ${ }^{36}$ : la collection de propositions $\beta$ est T-déductible de la collection de propositions $\alpha$ si et seulement si $\beta$ est déductible de $\alpha$ et que toutes les propositions qui figurent en tant que parties (directes) de $\alpha$ sont vraies ${ }^{37}$.

Dans la suite de l'article, « $\alpha$ » et « $\beta$ » sont utilisées comme variables (et parfois comme lettres schématiques) couvrant des collections de propositions vraies (vérités). L'énoncé suivant est-il vrai ?

(i) $\forall \alpha \forall \beta(\beta$ est T-déductible de $\alpha \rightarrow \alpha$ fonde $\beta$ )

Non. La fausseté de (i) peut se voir facilement à la lumière de certains exemples mentionnés dans ce qui précède. [Il fait plus chaud l'été que l'hiver] est T-déductible de [Les thermomètres qui fonctionnent bien montent plus haut l'été que l'hiver]. Les deux propositions sont vraies et la première est déductible de la seconde relativement aux représentations [été] et [hiver]. Mais la seconde proposition ne fonde pas (même seulement partiellement)

34. Cf. Bolzano, 1837, $\$ 200$.

35. Cf. Bolzano, 1837, $\$ 203$; Bolzano, Gesamtausgabe vol. 1/18 p. 22.

36. Bolzano considère lui aussi que cet aspect présente un intérêt décisif. Il soulève la question du lien entre ces relations principalement à la section $\$ 200$ de la Wissenschaftslehre.

37. Le cas échéant, la relation de déductibilité entre $\alpha$ et $\beta$ fait bien sûr en sorte que les propositions qui figurent en tant que parties (directes) de $\beta$ sont également vraies. Qu'est-ce qu'une partie directe d'une collection de propositions? Considérons la collection de [La terre est ronde] et [La terre est ronde $\rightarrow$ La lune est ronde]. [La terre est ronde] est une partie directe de cette collection. [La lune est ronde] en est une partie, mais pas une partie directe. Bolzano, Gesamtausgabe vol. $2 \mathrm{~A} 7$ p. $77 \mathrm{v}$. 
la première ${ }^{38}$. L'exemple illustre aussi une différence additionnelle : les deux propositions mentionnées sont T-déductibles dans un sens comme dans l'autre, mais elles ne se fondent certainement pas l'une l'autre. Il y a, de plus, une troisième différence, à savoir que chaque proposition vraie est T-déductible d'elle-même, alors qu'il n'y a rien de tel qui vaille en ce qui concerne la fondation.

Si (i) est fausse, qu'en est-il de sa converse?

(ii) $\forall \alpha \forall \beta$ ( $\alpha$ fonde $\beta \rightarrow \beta$ est T-déductible de $\alpha$ ).

Bolzano lui-même est indécis en ce qui concerne (ii). "Bien que [la vérité de (ii)] me semble plutôt probable, dit-il, je ne connais aucune preuve qui m'autoriserait à considérer la question close ${ }^{39}$. De plus, il tente de donner un exemple qui, selon lui, «semble prouver» que (ii) est fausse $e^{40}$.

Je n'aborderai pas cette question ici. Je passe outre cet exemple non seulement parce qu'il n'est pas facile de voir en quoi, exactement, il s'applique à (ii), mais aussi parce que Bolzano lui-même a des doutes à son endroit, et ce, principalement pour les deux raisons suivantes : d'une part, la question à savoir si (ii) est vraie ou fausse n'a pas nécessairement de rapport avec la question à savoir s'il y a une connexion conceptuelle entre [parce que] et le concept de T-déductibilité et, d'autre part, elle n'a pas, non plus, nécessairement rapport avec la question de savoir si la relation de fondation est une espèce de la relation de T-déductibilité (pour autant que la propriété d'être une espèce de propriété (property-kindhood) ne soit pas interprétée d'une façon purement extensionnelle) ${ }^{41}$. Même si (ii) est vraie, cela n'entraîne pas une connexion

38. Cf. Bolzano, 1837, $\mathbb{1 6 2}$. Mark Siebel a porté à mon attention une autre raison pour la fausseté de (i). Puisque la relation de déductibilité subsiste entre des collections de propositions et une représentation i même dans le cas où i n'apparaît pas dans une ou même toutes ces propositions, il s'ensuit que toute vérité est T-déductible de n'importe quelle collection de vérités à l'égard d'une représentation i si celle-ci n'apparaît ni dans la (ou les) prémisse(s), ni dans la conclusion. On pourrait éliminer ce problème additionnel en faisant appel à une notion qui pose les contraintes appropriées aux représentations pertinentes. Mais le principe analogue qu'on obtient en raffinant la notion de cette manière ne résiste pas à l'argument que je formule ici contre (i).

39. Bolzano, 1837, $\mathbb{} 200$.

40. Voir la fin de $\$ 200$. Mais (ii) serait vraisemblablement fausse pour une autre raison (que Bolzano ne mentionne pas) : malgré que le lien entre la fondation et la causalité doive être spécifié plus en détail —il semble peu probable que des (collections de) propositions qui « reflètent " des effets doivent être dans tous les cas déductibles de (collections de) propositions qui « reflètent» leurs causes.

41. Selon une interprétation non purement extensionnelle de la propriété d'être une espèce de propriété, le fait que tout ce qui satisfait la propriété n-adique F satisfait également la propriété n-adique $\mathrm{G}$ n'est pas suffisant pour que la propriété $\mathrm{F}$ soit un type spécial de propriété G. Il faut une connexion plus forte. Une conception non purement extensionnelle de la propriété d'être une espèce de propriété ne s'accorde pas avec l'usage que fait Bolzano du prédicat "la propriété $\mathrm{F}$ est une espèce de propriété $\mathrm{G}$ " (par exemple, à la section $\mathbb{} 200$ de la Wissenschaftslehre). Elle s'accorde cependant selon moi avec ce que nous disons ordinairement à propos des propriétés. 
conceptuelle entre les concepts concernés, et il ne s'ensuit pas non plus que la relation de fondement est une espèce de la relation de déductibilité.

On pourrait vouloir soutenir qu'on utilise parfois des énoncés de la forme 「p parce que q] pour dire que P s'ensuit de Q (ou, si c'est le concept de dérivabilité qui rend effectivement le concept usuel : que P est dérivable de Q). Il y a de tels usages, mais les énoncés sont-ils alors pris dans leur sens littéral? Dans l'affirmative, "parce que» serait, en définitive, équivoque. (Voir la fin de la section 3.) Mais, tel que nous l'avons vu, la signification de «P s'ensuit de $Q$ » peut difficilement être une signification littérale de " $p$ parce que $q$ " puisque la seconde implique que $\mathrm{P}$ et $\mathrm{Q}$ sont tous deux vrais, alors que ce n'est pas le cas pour la première. L'hypothèse dont on aurait besoin pour soutenir que "parce que " est ambigu consiste à dire qu'il y a un usage littéral de " $\mathrm{p}$ parce que q» qui signifie que $\mathrm{P}$ s'ensuit de $\mathrm{Q}$, et $\mathrm{P}$ et $\mathrm{Q}$ sont tous les deux vrais.

Les considérations des sections précédentes devraient avoir mis en lumière l'idée intuitive de fondation. Mais les résultats de cette entreprise, si elle vise à fournir une explication précise de ce concept, sont plutôt minces. Le seul résultat d'un quelconque intérêt pour quiconque tente de réduire le concept de fondation aux autres concepts est que la relation que met en jeu «parce que» est peut-être une espèce de la relation de T-déductibilité. Mais même si nous pouvions le démontrer, cela ne nous éclairerait pas davantage sur ce concept. Il semble donc pertinent de chercher une explication indépendante. Et c'est ce que fait Bolzano.

\section{La théorie de Bolzano : remarques préliminaires}

J'en viens maintenant à la théorie de la fondation propre à Bolzano. Ce dernier distingue deux concepts : la fondation immédiate et la fondation médiate. La fondation immédiate est essentiellement une relation bijective entre deux objets uniques. L'idée intuitive de fondation médiate est qu'une collection de vérités est un fondement médiat d'une autre collection de vérités $\beta$ si et seulement s'il y a une "chaîne » d'instances de la relation de fondation immédiate (au moins partielle) qui va de $\alpha$ à $\beta$, via un autre élément ${ }^{42}$.

Bolzano explique l'idée intuitive de fondation en invoquant les deux concepts. À mon avis, il faut comprendre sa démarche comme consistant à fournir des définitions implicites (partielles) des concepts de fondation immédiate et médiate. À cet effet, Bolzano pose, sans toutefois procéder dans un ordre très systématique, une série de propositions qui ont pour but de caractériser

42. Les termes «fondement immédiat» et «fondement médiat» révèlent à mon sens le point central de la relation entre les deux concepts mieux que n'importe quelle autre paire de termes. Malgré cela, Bolzano n'utilise pas ces termes, parce qu'il considère que ce que j'appelle "fondements (conséquences) médiats» ne sont pas vraiment des fondements (conséquences) dans le sens propre du terme. (Voir $\mathbb{S} \mathbb{2 1 3}$, 217). Je ne crois pas que Bolzano ait raison si notre compréhension de «fondement» et «conséquence» doit être dérivée de l'usage ordinaire de "parce que ». Nous y reviendrons à la fin de l'article. 
les concepts en question. Ces propositions peuvent cependant être réduites à trois clauses définitionnelles implicites (deux pour le concept de fondation immédiate, une pour le concept de fondation médiate). Ainsi, la théorie de Bolzano peut être rendue par trois axiomes qui fournissent des définitions implicites (connexes) des concepts de fondation immédiate et médiate. Les deux premiers axiomes (qui caractérisent la fondation immédiate) sont spécifiés à la section 6, le troisième (qui caractérise la fondation médiate) à la section 7 .

On m'a fait remarquer qu'il est peut-être erroné de reconstituer la méthode de Bolzano comme si elle fournissait des définitions implicites. On considère habituellement qu'une définition implicite consiste à stipuler que certains énoncés sont (ou devraient être) vraies. Mais, pour poursuivre avec cette objection, les porteurs de vérité et fausseté primitifs sont chez Bolzano, des propositions, et non des énoncés. Il semble donc qu'il faille considérer une définition implicite comme une stipulation à l'effet que certaines propositions sont (ou devraient être) vraies. Mais peut-on réellement stipuler qu'une proposition est (ou devrait être) vraie?

La réponse à cette objection est selon moi la suivante : bien entendu, on ne peut pas d'abord fixer une proposition (quel que soit le moyen utilisé) et ensuite stipuler que cette proposition est (ou devrait être) vraie : la proposition pourrait refuser d'obéir. Mais lorsqu'il s'agit de donner une signification à un terme, il est permis de faire la chose suivante, à savoir : stipuler à propos de certains énoncés contenant ce terme que, peu importe les propositions qu'ils expriment, elles sont (ou devraient être) vraies. On peut ainsi restreindre la signification d'un terme (de manière plus ou moins forte). En ceci consiste, d'après moi, la démarche de Bolzano (du moins si l'on prend également en compte que ses stipulations ne sont non pas arbitraires, mais qu'elles visent à exprimer notre concept ordinaire de [parce que]).

Pour formuler la théorie de Bolzano, il nous faut éclaircir la distinction entre fondements et conséquences partiels et complets. Quelques remarques générales à propos de son usage de "collection » et "partie " s'imposent également. Bolzano assume que les expressions telles que « $\mathrm{a}$, b et $\mathrm{c}$ » (où « $\mathrm{a}$ », « $\mathrm{b} »$ et $« \mathrm{c} »$ sont des termes singuliers) référent à des collections. C'est selon moi le principe central de la signification qu'il attache à «collection ". Si a, b et c sont tous des F, alors la collection consistant en a, b et c (et rien d'autre) est une collection de F. Dans le présent contexte, Bolzano parle fréquemment de collections de propositions.

Bolzano dirait que $\mathrm{a}, \mathrm{b}$ et $\mathrm{c}$ sont toutes des parties de la collection des a, b et c. Mais il est important de noter que cet usage du mot «partie» ne s'accorde pas - quoiqu'il soit tout à fait naturel — avec la méréologie contemporaine. Il en est ainsi parce que la relation que Bolzano dénote par «partie» n'est pas transitive. «Il est vrai seulement de collections d'un type spécial que «les parties d'une partie sont parties du tout ", comme le disent certains ${ }^{43}$. Le

43. Bolzano, 1837, $\mathbb{S} 83.2$. 
roi et ses ministres, pour reprendre un exemple de Bolzano, font partie de l'État, les bras du roi font partie du roi, mais les bras du roi ne font pas partie de l'État (selon le sens que Bolzano attribue à «partie») ${ }^{44}$.

Dans le présent contexte, nous nous intéressons aux collections de vérités (en tant que termes des relations de fondation). Il faudra, au moins pour les fins de la commodité, assumer qu'il y a des collections qui n'ont qu'une seule partie $^{45}$. Comme Bolzano, je présupposerai également deux principes qui valent pour toutes les collections de vérités $\alpha$, $\beta$, et $\gamma$ : la collection des $\alpha$ et $\beta=$ la collection des $\beta$ et $\alpha$. La collection des $(\alpha$ et $\beta$ ) et $\gamma=$ la collection des $\alpha$, $\beta$, et $\gamma$. Le second principe implique que la relation de partie est transitive pour autant que nous restreignions le domaine du discours aux collections de vérités. Ainsi la différence sus-mentionnée entre le concept de partie de Bolzano et celui de la méréologie standard n'importe pas en ce qui concerne ici.

Les collections de Bolzano héritent du statut ontologique de leurs parties. La collection de mes copies des quatre volumes de la Wissenschaftslehre d'une part, est aussi concrète que le sont les livres individuellement : son poids est la somme des poids de chacun des volumes, son emplacement est celui des quatre volumes (c'est un «objet dispersé »), et ainsi de suite. D’autre part, considérons l'objet qu'on pourrait appeler «la Wissenschaftslehre de Bolzano en soi ", c'est-à-dire, une certaine collection de propositions ordonnées d'une certaine manière. Cette collection est aussi abstraite que n'importe laquelle des propositions qui en font partie : elle n'a ni propriétés spatiales, ni propriétés temporelles.

Alors que ces propriétés distinguent clairement les collections des ensembles, elles sont partagées par les sommes ou fusions méréologiques ${ }^{46}$. Les collections ne sont-elles alors rien d'autre que des sommes méréologiques? Le fait que Bolzano n'entende pas par "partie» la même chose que les méréologues semble révoquer cette possibilité. D’un autre côté, il est important de noter que le concept bolzanien de collection ne dépend pas du concept qu'il exprime par "partie». (Ce qui, soit dit en passant, fait contraste avec la méréologie où le concept de somme est dérivé du concept de partie.) À mon avis, la bonne façon d'introduire les concepts de Bolzano est celle que j'ai adoptée ici : d'abord, introduire [collection] en faisant appel à des formules telles que "a,b, et c », et ensuite introduire [partie] en faisant appel à [collection]. Ainsi, le fait que les concepts de partie qu'elles impliquent respectivement soient différents n'empêche pas nécessairement les collections d'être

44. Cf. Bolzano, Gesamtausgabe vol. $2 \mathrm{~A} 7$ p. $77 \mathrm{v}$.

45. Ce principe ne concorde pas avec l'introduction officielle du terme «collection» à la section $\ 82$ de la Wissenschaftslehre, où Bolzano suggère que chaque collection de $\mathrm{F}$ a au moins deux F comme parties. Néanmoins, cela s'accorde avec l'usage que Bolzano fait du terme à plusieurs endroits. Le fait de s'en tenir au terme tel que Bolzano l'introduit aurait pour résultat de compliquer plusieurs formulations, mais cela ne changerait rien de substantiel.

46. Cf. Simons, 1987. 
des sommes méréologiques (bien que cela empêche que le concept de collection soit le concept méréologique de somme) ${ }^{47}$.

Il faut introduire ici quelques symboles : $« \alpha \Rightarrow \beta »$ doit être lu comme $« \alpha$ est le fondement objectif complet immédiat de $\beta$, et $\beta$ est la conséquence objective complète et immédiate de $\alpha »$. Notez que « $\Rightarrow »$ connecte des termes singuliers, et non des énoncés. Tel que je le formule ici, le but de la théorie de Bolzano (ou au moins l'un d'eux) est de fixer la signification de « $\Rightarrow »$ par une clause définitionnelle implicite appropriée de façon à exprimer autant que possible la signification ordinaire de "parce que». Les symboles suivants seront également utiles :

Définition de $\Rightarrow: \forall \alpha \forall \beta\left[\alpha_{<} \Rightarrow \beta\right.$ ssi $\left.\exists \gamma(\gamma \Rightarrow \beta \& \alpha<\gamma)\right]$.

Définition de $\Rightarrow_{<<}: \forall \alpha \forall \beta\left[\alpha \Rightarrow_{<<} \beta\right.$ ssi $\left.\exists \gamma(\alpha \Rightarrow \gamma \& \beta \leq \gamma)\right]$.

« $\alpha<\beta$ » est l'abréviation de « $\alpha$ est une partie de $\beta$ ou $\alpha=\beta$ ». « $\alpha_{<<} \beta$ » est l'abréviation de « $\alpha$ est une partie de $\beta$ mais $\alpha \neq \beta »{ }^{48}$. Donc $« \alpha<\beta$ « doit être lue comme « $\alpha$ est un fondement partiel ou le fondement immédiat complet de $\beta$ », tandis que $« \alpha \Rightarrow{ }_{<<} \beta$ » doit être lu comme « $\beta$ est une conséquence partielle mais non la conséquence immédiate complète de $\alpha$ ». Le sens de l'asymétrie entre ces définitions se clarifiera par la suite.

\section{La fondation immédiate}

Tel que le caractérise Bolzano, le concept de fondation immédiate peut être rendu par deux axiomes. En ce qui concerne la proposition qui tient lieu de mon premier axiome, Bolzano ${ }^{49}$ fait explicitement appel à Aristote qui, dans le livre $\Theta$ de la Métaphysique, dit ceci :

Ce n'est pas parce que nous pensons d'une manière vraie que tu es blanc, que tu es blanc, mais c'est parce que tu es blanc, qu'en disant que tu l'es, nous disons la vérité ${ }^{50}$.

Bolzano interprète ce passage comme énonçant une thèse sur une relation entre propositions. Il considère qu'Aristote dit en fait ceci : $\left(\mathrm{A}_{1}\right)$ Ce n'est pas que [Il est vrai que tu es blanc] fonde (partiellement) [tu es blanc], mais plutôt que : $\left(\mathrm{A}_{2}\right)$ [tu es blanc] fonde (partiellement) [Il est vrai que tu es blanc]. La Wissenschaftslehre contient à la fois des versions généralisées et plus spécifiques de $\mathrm{A}_{1}$ et $\mathrm{A}_{2}{ }^{51}$. Sa version de $\mathrm{A}_{1}$ fait figure de prémisse dans son argu-

47. Krickel (1995) soutient que les collections de Bolzano doivent plutôt être vues comme des sommes méréologiques; Simons (1997) argumente contre cette position, tandis que Behboud (1997) tente d'en défendre une version.

48. La relation entre ce qui est dénoté par «<» et «<<» dans la méréologie est similaire à la relation entre ce qui est dénoté par «s» ( «est plus petit que ou identique à») et «<» ( est plus petit que») en arithmétique. Voir aussi Simons, 1987, p. 10s.

49. Bolzano, 1837, $\mathbb{S} 198$, note.

50. Aristote, Métaphysique, livre $\Theta 10: 1051$ b 6-9 (trad. fr. tome II, p. 522).

51. La section $\mathbb{} 206$ de la Wissenschaftslehre contient une version de $\mathrm{A}_{2}$ qui s'applique aux collections de propositions. 
ment pour l'asymétrie de la fondation immédiate (qui sera bientôt cité). Voici la formulation bolzanienne de $\mathrm{A}_{2}$ :

Quelle que soit la vérité $\mathrm{A}$, la vérité «que la proposition A est vraie» en est une conséquence véritable; et cette conséquence ne nécessite certes nullement comme fondement (Begründung) une deuxième vérité en plus de $\mathrm{A}$, ce qui fait de cette dernière son fondement (Grund) complet $^{52}$.

À l'aide des symboles introduits dans la section précédente, nous pouvons formuler la version bolzanienne de l'affirmation d'Aristote comme suit (je rappelle ici que j'utilise $\langle\alpha », " \beta »,\langle\gamma »$...comme variables de collections de vérités) :

\section{Premier axiome pour $« \Longrightarrow »$ (principe d'Aristote)}

(i) $\forall \alpha \neg\left([\alpha \text { est vraie }]_{<} \Rightarrow \alpha\right)$.

(ii) $\forall \alpha\left(\alpha \Rightarrow \Rightarrow_{<<}[\alpha \text { est vraie }]\right)^{53}$.

L'intuition derrière la clause (i) est claire : il semble déraisonnable d'assumer que certaines collections de vérités $\alpha$ peuvent être fondées, partiellement ou même complètement, sur la vérité [ $\alpha$ est vraie]. Intuitivement, il semble tout aussi clair que [ $\alpha$ est vraie] est toujours une des conséquences de $\alpha$. Il pourra de plus sembler surprenant que Bolzano considère qu'il est intuitivement clair que [ $\alpha$ est vraie] n'est jamais la seule conséquence (partielle) de $\alpha$, ce qu'il assume d'ailleurs explicitement :

À la conséquence complète de certaines vérités $\mathrm{A}, \mathrm{B}, \mathrm{C}, \mathrm{D}, \ldots$ appartient, entre autres aussi la vérité «que toutes les propositions $\mathrm{A}, \mathrm{B}, \mathrm{C}, \mathrm{D}, \ldots$ sont vraies». Mais il s'agit d'une conséquence (à savoir une conséquence partielle) que n'a manifestement aucune autre collection [de vérités] que celle-là ${ }^{54}$.

Bolzano mentionne également de manière explicite un théorème qui résulte visiblement de la clause (ii) du principe d'Aristote, et qui affirme que :

[...] même dans les cas où le fondement est une vérité individuelle, il y a toujours plusieurs vérités qui, prises collectivement, peuvent être considérées comme sa conséquence ${ }^{55}$.

Ce qui se traduit sous forme symbolique par :

Théorème $1: \forall \alpha \forall \beta\left(\alpha \Rightarrow \beta \rightarrow \exists \mathrm{P} \exists \mathrm{Q}\left(\mathrm{P}_{<<} \beta \& \mathrm{Q}_{<<} \beta\right)\right)$ (complexité de la conséquence).

L'idée de Bolzano semble être que toute vérité ou collection de vérités $\alpha$ fonde, pour ainsi dire, «quelque chose de substantiel » et, en plus de cela, la vérité $[\alpha$ est vraie].

52. Bolzano, 1837, $\mathbb{\int} 205.1 ; \mathrm{Si} \alpha$ est la collection de propositions A, B, et C, «[ $\alpha$ est vraie] » est alors l'abréviation de «[A, B, et C sont vraies] ». Voir la notation de Bolzano à la section $\mathbb{2} 206$ de la Wissenschaftslehre.

53. Cf. Bolzano, 1837, $\mathbb{S}$ 205.1, 206, 209, 212, 214.

54. Ibid., $\mathbb{} 206$.

55. Ibid., $\mathbb{\$} 205.2$. 
Bolzano prend pour acquis les deux volets du principe d'Aristote à plusieurs endroits ${ }^{56}$. Cependant, la Wissenschaftslehre ne contient pas de section qui y soit spécifiquement consacrée (même seulement en partie); Bolzano ne fournit aucun argument pour le principe d'Aristote. Ainsi il semble que, conformément à ma reconstruction, il aurait accepté de le considérer comme un principe primitif.

Il faut toutefois également mentionner qu'il y a une autre interprétation possible d'Aristote. Selon cette seconde interprétation, Aristote n'exprime pas dans le passage cité une thèse à propos d'une relation entre propositions mais soutient plutôt une thèse à propos des propositions et de leurs vérifacteurs - où un vérifacteur n'est pas une proposition mais quelque chose «dans le monde» (un état de choses, un accident individuel ou quoi que ce soit d'autre $)^{57}$. Bolzano n'attribue pas à Aristote la thèse de la «vérifaction » dans le sens qui vient d'être décrit, et refuse lui-même d'y adhérer. Au contraire, il croit qu'il n'y a rien de tel que la relation de "vérifaction » :

Une chose $\mathrm{X}$ a-t-elle la propriété $\mathrm{x}$ parce que la proposition $\mathrm{X}$ qui a la propriété $\mathrm{x}$ est vraie, ou à l'inverse, cette proposition est-elle bien plutôt vraie parce que la chose $\mathrm{X}$ possède cette propriété ? La bonne réponse, à mon avis, est : ni l'une, ni l'autre. Le fondement de la vérité d'une proposition se trouve, si la vérité de cette proposition a un fondement (Grund), dans une autre vérité, et non dans la chose sur quoi elle porte. Et on peut encore moins dire que la raison pour laquelle $\mathrm{X}$ a la propriété $\mathrm{x}$ repose dans la vérité que $\mathrm{X}$ a la propriété $\mathrm{x}$. Si X est effectivement une chose réelle, alors il ne peut y avoir de fondement en vertu duquel elle a la propriété $\mathrm{x}$, mais il peut $\mathrm{y}$ avoir une cause qui explique qu'elle a la propriété $\mathrm{x}$, et cette cause repose dans une autre chose ${ }^{58}$.

En niant que « la proposition $\mathrm{X}$ a la propriété $\mathrm{x}$ est vraie [...] parce que la chose X a cette propriété », Bolzano ne contredit pas le principe d'Aristote, puisque (tel que le montre la suite du passage) Bolzano a ici apparemment à l'esprit l'interprétation "vérificationniste» de ce principe.

Il vaut la peine de souligner que Bolzano est ici en désaccord avec plusieurs réalistes autrichiens (et australiens!) qui soutiennent qu'il y a une relation de vérifaction entre les propositions et certaines choses (ou certains types de choses) «dans le monde ». Cela est vrai même si ces derniers ne défendent habituellement pas la position sur laquelle se penche Bolzano dans le passage cité, c'est-à-dire même s'ils n'identifient pas le vérifacteur d'une proposition à la chose sur laquelle porte la proposition (mais avec des états de chose, comme Armstrong, ou avec des moments individuels, comme Mulligan, Simons, et Smith $)^{59}$. Ce détail pourra paraître d'autant plus intéressant que

56. Sur le concept de vérifacteur, voir Mulligan, Smith et Simons, 1984. La différence, de même que les relations entre ces deux interprétations possibles d'Aristote sont expliquées en détail dans Künne, à paraître, ch. 3.5.

57. Voir Armstrong, 1997; Mulligan, Smith et Simons, 1984.

58. Bolzano, Gesamtausgabe vol. 2 A 12/2, p. 60.

59. Cf. Bolzano, 1837, $\mathbb{} 206$. 
la tradition réaliste autrichienne fut, à maints égards, fondée par Bolzano lui-même.

Bolzano considère que la fondation immédiate est une relation bijective entre deux objets uniques dans le sens où chaque fondement immédiat (complet) a son unique conséquence immédiate (complète), et chaque conséquence immédiate (complète) a son unique fondement immédiat (complet). Ce principe constitue le second axiome de la fondation immédiate.

Second axiome pour $« \Rightarrow »: \forall \alpha \forall \beta(\alpha \Rightarrow \beta \rightarrow \forall \gamma((\gamma \Rightarrow \beta \rightarrow \gamma=\alpha) \&(\alpha \Rightarrow \gamma$ $\rightarrow \gamma=\beta))$ ) (unicité).

Bolzano soutient que ce principe devient extrêmement plausible dès qu'on concède que, à toutes les fois qu'une collection de propositions semble avoir plus d'un fondement (conséquence) immédiat, les candidats à ce titre doivent plutôt être considérés comme différentes parties de son unique fondement (conséquence) immédiat ${ }^{60}$.

Cependant, on pourrait avancer que l'unicité de la relation de fondation immédiate est, intuitivement, inappropriée pour la raison suivante. Considérons une proposition disjonctive $[\mathrm{PvQ}]$ dont les membres sont tous les deux vrais. On pourrait être tenté de dire que $\mathrm{P}$ et $\mathrm{Q}$, indépendamment l'un de l'autre, sont les fondements complets de [PvQ] (ou quelque collection plus large incluant $[\mathrm{PvQ}])$. Mais, Bolzano serait tenu de dire que $[\mathrm{PvQ}]$ n'a qu'un seul fondement, à savoir la collection de $\mathrm{P}$ et $\mathrm{Q}$.

On pourrait être tenté, mais, bien entendu, personne n'est tenu de voir les choses sous cet angle. Quoi qu'il en soit, on est en droit d'exiger plus qu'une simple intuition en ce qui concerne la question des fondements multiples. Et Bolzano a effectivement un argument pour appuyer sa conclusion, à savoir qu'il n'y a pas une chose telle que la fondation multiple. Son argument est, bien entendu, un argument de plausibilité. (Comment pourrait-on argumenter autrement pour un axiome?) En formulant son argument, Bolzano nous rappelle que les conséquences (complètes) ne consistent jamais en une seule vérité (notre théorème 1) - contrairement à ce qui est suggéré par l'exemple de la disjonction. Il écrit, en rapport avec des exemples analogues à ce dernier :

[...] considérés attentivement, ces exemples ne montrent rien d'autre que différents fondements ont parfois des conséquences partielles (Theilfolge) communes; ils ne montrent pas que la conséquence complète est la même. À la conséquence complète de certaines vérités $\mathrm{A}, \mathrm{B}, \mathrm{C}, \mathrm{D}, \ldots$ appartient, entre autres, la vérité «que les propositions $\mathrm{A}, \mathrm{B}, \mathrm{C}, \mathrm{D}, \ldots$ sont toutes vraies". Mais c'est là une conséquence (à savoir une conséquence partielle) qu'aucune autre collection de vérités ne possède, à part celle-là. Il devient alors clair que chaque fondement propre a une conséquence qui, au moins à l'égard de certaines de ses parties (in gewissen Stücken) a, elle aussi, quelque chose de propre ${ }^{61}$.

60. Bolzano fait ici écho à la première partie du principe d'Aristote, c'est-à-dire $A_{1}$. À noter que $\mathrm{A}_{2}$ fait également ici figure de prémisse.

61. Bolzano, 1837, \206. 
Appliquée à notre exemple de la disjonction, l'idée est que la conséquence de $\mathrm{P}$ - selon le principe d'Aristote - comprend la vérité [P est vraie], mais elle ne contient pas — plausiblement — la vérité [Q est vraie],

tandis que

la conséquence de Q - selon le principe d'Aristote - comprend la vérité [Q est vraie], mais — en toute plausibilité — elle ne contient pas la vérité [P est vraie].

La conséquence (complète) de $\mathrm{P}$ est par conséquent différente de la conséquence (complète) de $\mathrm{Q}$. Ainsi, on ne peut pas dire que $[\mathrm{P} \vee \mathrm{Q}]$ se fonde à la fois sur $\mathrm{P}$ et $\mathrm{Q}$ - du moins pas au sens du «fondée » qui nous intéresse ici : ni $[\mathrm{PvQ}]$ ni quelque collection de vérités la contenant n'est la conséquence complète de $\mathrm{P}$ et $\mathrm{Q}$; et ces dernières n'en sont pas le fondement complet. Cela est exclut puisque, simplement, $\mathrm{P}$ et $\mathrm{Q}$ ont des conséquences (complètes) différentes.

Comme que je l'ai mentionné, il s'agit là d'un argument de plausibilité. Il s'appuie (en plus du principe d'Aristote) sur l'hypothèse plausible que de vérités $\mathrm{P}$ et $\mathrm{Q}$ ne sont jamais toutes deux reliées comme fondements complets d'une "grosse » conséquence qui serait leur seule conséquence complète et qui comprendrait les deux vérités : [P est vraie] et [Q est vraie]

J'en viens maintenant à dériver quelques théorèmes additionnels. La fondation immédiate est une relation asymétrique : il n'y a pas de collections de vérités qui se fondent immédiatement les unes les autres de façon mutuelle.

Théorème $2: \forall \alpha \forall \beta(\alpha \Rightarrow \beta \rightarrow \neg(\beta \Rightarrow \alpha))$ (asymétrie).

On retrouve l'idée d'une preuve indirecte pour le théorème 2 dans le passage suivant :

Il est clair que si les vérités $\mathrm{A}, \mathrm{B}, \mathrm{C}, \ldots$ qui constituent le fondement complet des vérité $\mathrm{M}, \mathrm{N}, \mathrm{O}, \ldots[\ldots]$ elles ne peuvent en être également la conséquence, car à cette conséquence complète $\mathrm{M}, \mathrm{N}, \mathrm{O}, \ldots$ appartient aussi la proposition que les $\mathrm{A}, \mathrm{B}, \mathrm{C}, \ldots$ sont toutes vraies; mais cette proposition devrait alors elle aussi être considérée être le fondement partiel des vérités $\mathrm{A}, \mathrm{B}, \mathrm{C}, \ldots$, ce qui est absurde ${ }^{62}$.

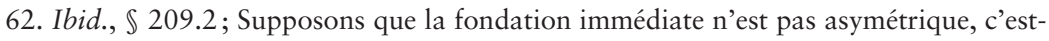
à-dire qu'il y a des collections de vérités $A$ et $B$ telles que (1) $A \Rightarrow B \& B \Rightarrow A$ est valide. Ceci implique de manière triviale que $\mathrm{A}$ est une collection de vérités. Il s'ensuit alors par la clause (ii) du principe d'Aristote que (2) A $\Rightarrow_{<<}$[A est vrai]. De (1), (2), et (Df. $\Rightarrow_{<<}$), il s'ensuit que (3) [A est vrai $]_{<<} B$. D’un autre côté, il découle de (1) et de la clause (i) du principe d'Aristote que (4) $\neg([$ A est vrai] $\Rightarrow$ A). De (1), (4), (Df. $\Rightarrow)$, et de l'axiome 2 il s'ensuit que $(5) \neg([$ A est vrai $]<$ B). Mais, d'après le principe de la logique des parties selon lequel $\forall \mathrm{x}, \mathrm{y}\left(\mathrm{x}_{<<} \mathrm{y} \rightarrow \mathrm{x}<\mathrm{y}\right),(5)$ est inconsistant avec (3). Puisque (1) est la seule hypothèse que nous ayons faite (le principe d'Aristote mis à part), il faut la rejeter : (6) $\neg(A \Rightarrow B \& B \Rightarrow A)$. Étant donné que n'avons pas fait d'autre hypothèse à propos de $\mathrm{A}$ et $\mathrm{B}$; il est donc aussi justifié de généraliser (6) : (7) $\forall \alpha \forall \beta \neg(\alpha \Rightarrow \beta \&$ $\beta \Rightarrow \alpha)$ qui est l'équivalent du théorème 2 . 
La fondation immédiate est également irréflexive : rien n'est son propre fondement complet (ou sa propre conséquence complète).

Théorème $3: \forall \alpha \neg(\alpha \Rightarrow \alpha)$ (irréflexivité).

Cela peut, encore une fois, être prouvé indirectement. Supposons que la relation de fondation immédiate n'est pas réflexive. Il y aurait alors une collection de vérités $A$, telle que $A \Rightarrow A$. Mais, en vertu du théorème 2 (asymétrie), il s'ensuit une contradiction, à savoir que $A \Rightarrow A \& \neg(A \Rightarrow A)$. Ainsi, la fondation immédiate est irréflexive. Bolzano lui-même ne déduit pas l'irréflexivité de l'asymétrie; il considère que l'irréflexivité est une caractéristique triviale du concept de fondation immédiate et ne donne aucune raison additionnelle ${ }^{63}$.

Bolzano note que plusieurs philosophes semblent nier l'irréflexivité de la relation de fondation immédiate. Ces philosophes disent à propos de certaines propositions «qu'elles sont fondées complètement en elles-mêmes ». Bolzano soutient que ces philosophes ne nient pas réellement que la relation de fondation immédiate est irréflexive, mais qu'ils parlent plutôt d'une façon non rigoureuse $^{64}$. Selon Bolzano, on veut dire, tantôt que ces propositions n'ont pas de fondement du tout (ce que Bolzano soutient également, comme nous le verrons dans ce qui suit), tantôt que ces propositions sont fondées seulement dans les propriétés (Beschaffenheiten) des concepts qui s'y retrouvent. Si on considère comme fondements non pas ces propriétés en elles-mêmes mais les propositions vraies qui les décrivent, cela semble être vrai. Mais, poursuit Bolzano, le cas échéant, la vérité en question n'est pas son propre fondement; ce fondement est bien plutôt la collection de vérités décrivant les propriétés pertinentes.

Qu'en est-il de la transitivité ? Cela peut sembler surprenant, mais la fondation immédiate est clairement intransitive.

Théorème $4: \forall \alpha \forall \beta \forall \gamma((\alpha \Rightarrow \beta \& \beta \Rightarrow \gamma) \rightarrow \neg(\alpha \Rightarrow \gamma))$ (intransitivité).

L'argument de Bolzano à cet égard est simple et convaincant ${ }^{65}$. Il se résume à dire que si la fondation immédiate n'était pas intransitive, il y aurait des collections de vérités $A, B, C$ telles que $A \Rightarrow B \& B \Rightarrow C \& A \Rightarrow C$. Mais cela ne peut être le cas, puisque $\mathrm{C}$ devrait alors avoir deux fondements immédiats (complets) différents, ce qui est proscrit par l'axiome 2 (unicité).

Bolzano reconnaît que l'intransitivité de la fondation immédiate pourra être considérée contre-intuitive, du moins pour quiconque accepte - comme lui - qu'il y a une connexion étroite entre la fondation et la relation de causalité du type suggéré par le "modèle à deux niveaux " ${ }^{66}$. Selon ce modèle, les faits causaux sont reflétés par une relation de fondation immédiate entre les propositions vraies qui énonce les causes et effets en question. Or, si le modèle à deux niveaux est correct il semble que, si la relation de fondation immédiate

63. Cf. Bolzano, 1837, $\$ 204$.

64. Cf. Ibid., $\mathbb{S} 204$, note.

65. Cf. Ibid., $\$ 213$.

66. J'ai fourni une esquisse du modèle à deux niveaux à la section 3. 
est intransitive, la relation de causalité doit l'être également ${ }^{67}$. Or, intuitivement, ce n'est pas le cas. Dans le langage ordinaire, il ne semble y avoir aucun problème à appeler la cause d'une cause de $\mathrm{x}$ une "cause de $\mathrm{x}$ ». Supposons qu'une maison a été détruite par un éboulis qui est lui-même causé par le fait que Max a lancé une pierre. Dans ce cas, l'affirmation que la pierre lancée par Max a causé la destruction de la maison semble être aussi vrai que celle disant que l'éboulis a causé la destruction de la maison.

Bolzano concède ce point ${ }^{68}$, mais je crois cependant qu'il se défend inutilement. Il est important de se rappeler que nous nous occupons ici exclusivement du concept de fondation immédiate pour le moment. Le fait que la relation de fondation immédiate soit intransitive n'empêche toutefois nullement qu'il en soit autrement de la relation de fondation médiate. La fondation médiate est d'ailleurs bel et bien transitive; c'est là l'une des différences majeures entre les deux relations. Ainsi, Bolzano aurait facilement pu renvoyer cette interprétation (de cet aspect) de notre usage habituel de «parce que» au concept de fondation médiate; c'est l'idée que je défendrai dans la prochaine section.

Du principe d'Aristote (et de la définition de $« \Rightarrow_{<<}$), il s'ensuit manifestement que chaque collection de propositions est le fondement immédiat de quelque chose d'autre :

\section{Théorème $5: \forall \alpha \exists \beta(\alpha \Rightarrow \beta)^{69}$}

Notez que, malgré cela, on ne peut dériver des deux axiomes que toute collection de vérités est une conséquence de quelque chose d'autre. En fait, à la section $\$ 214$ de la Wissenschaftslehre, Bolzano nie que toute collection de vérités est une conséquence. Ses arguments pour l'existence de vérités primitives (Grundwahrheiten) ne sont pas basés sur des caractéristiques additionnelles du concept de fondation (qui ne seraient pas, quant à eux, exprimées par les axiomes); ils sont complètement indépendants ${ }^{70}$. Cela se manifeste dans le fait que nos deux axiomes n'excluent, pas plus qu'ils n'impliquent, l'existence de vérités primitives.

Un autre résultat de la conception bolzanienne de la fondation immédiate est qu'il y a des collections de vérités qui sont à la fois des fondements immédiats (partiels ou complets) et des conséquences immédiates (partielles ou complètes $)^{71}$. À partir de nos clauses définitionnelles implicites primitives, nous pouvons dériver

Théorème $6: \forall \alpha \exists \beta \exists \gamma(\alpha \Rightarrow \beta \& \beta \Rightarrow \gamma)^{72}$.

67. Notez que l'implication reste valide même lorsqu'on ne présuppose pas la thèse particulière de Bolzano à propos de la signification de «cause » (voir section 3).

68. Cf. Bolzano, 1837, $\mathbb{2} 213$, note.

69. Cf. Ibid., $\mathbb{S} 214$.

70. Cf.Ibid., $\mathbb{S}$ 214, 221.3.

71. Cf. Ibid., $\mathbb{} 208$.

72. Supposons que a est une collection arbitraire de vérités. Selon le théorème 5 , il y aurait une autre collection de vérités $\beta$ telles que $\alpha \Rightarrow \beta$. Il en est de même pour b elle-même : il 
Afin de dériver qu'il y a en fait des collections de vérités $\beta$ et $\gamma$ telles que $(\alpha \Rightarrow \beta \& \beta \Rightarrow \gamma)$, nous devons supposer qu'il y a des propositions vraies. Pour Bolzano, cette hypothèse n'est pas problématique : c'est un théorème de sa Fundamentallehre, la première partie de la Wissenschaftslehre ${ }^{73}$.

Ainsi il pourrait y avoir des collections de vérités A, B, C, D ... telles que A est le fondement immédiat de $\mathrm{B}$, B le fondement immédiat de $\mathrm{C}, \mathrm{C}$ le fondement immédiat de $\mathrm{D}$, et ainsi de suite. Mais puisque la relation de fondation immédiate est intransitive, A n'est ni le fondement immédiat partiel, ni le fondement immédiat complet de D. Mais A est un fondement médiat de D. À la section $\$ 216$ de la Wissenschaftslehre, Bolzano élargit le champ de son enquête en tournant son attention vers la relation de fondation médiate ${ }^{74}$.

\section{Fondation médiate}

J'appelle la procédure par laquelle quelqu'un qui cherche le fondement d'une certaine vérité $\mathrm{M}$, et la trouve dans la vérité $\mathrm{L}$ ou dans la collection de vérités $\mathrm{I}, \mathrm{K}, \mathrm{L}, \ldots$, cherche ensuite le fondement ou les fondements de l'une ou de plusieurs de ces vérités, et ainsi de suite [...], remontée de la conséquence au fondement ${ }^{75}$.

Les fondements médiats d'une vérité sont les vérités et collections de vérités auxquelles conduit la remontée de la conséquence au fondement. L'idée intuitive (tel que mentionné à la section 5.) est la suivante : une collection de vérités $\alpha$ est le fondement médiat d'une autre collection de vérités $\beta$ si et seulement s'il y a une "chaîne" d'instances de la relation de fondation immédiate (complète ou partielle) de $\alpha$, via quelques autres éléments, à $\beta^{76}$. Pour les fin de la clarté, j'utilise $« \alpha \sqrt{ } \beta$ » comme abbéviation pour « $\alpha$ est le fondement

doit y avoir un $\mathrm{g}$ tel que $\beta \Rightarrow \gamma$. Ainsi, l'existence d'une collection arbitraire de vérités a fait en sorte que $\exists \beta \exists \gamma(\alpha \Rightarrow \beta \& \beta \Rightarrow \gamma)$. D’où découle le théorème 6.73. Cf. Bolzano, 1837, $\mathbb{S} 31$.

74. Bolzano caractérise la relation de fondation immédiate par d'autres propositions qu'on doit mentionner en passant. Les principes formulés à la section $\mathbb{S} 207$ de la Wissenschaftslehre découlent de l'axiome 2 : à savoir, que la conséquence d'une partie propre d'une certaine collection de vérités n'est jamais la conséquence de cette collection elle-même $\left(\forall \alpha \forall \beta \forall \gamma\left(\left(\alpha \Rightarrow \beta \& \alpha_{<<} \gamma\right) \rightarrow \neg \gamma \Rightarrow \beta\right)\right)$, et que le fondement immédiat d'une partie propre d'une certaine collection de vérités n'est jamais le fondement immédiat de cette collection elle-même $\left(\forall \alpha \forall \beta \forall \gamma\left(\left(\alpha \Rightarrow \beta \& b \Rightarrow b_{<} \gamma\right) \rightarrow \neg \alpha \Rightarrow \gamma\right)\right)$. À la section $\mathbb{} 212$, Bolzano suppose qu'il y a des collections de vérités qui sont reliées comme fondement et conséquence immédiats et, de plus, qui "peuvent être combinés en tant que parties pour entraîner une nouvelle conséquence ». En référence au théorème 5 , il est facile de montrer qu'il y a non seulement de telles collections de vérités, mais que ceci vaut pour toute collection de vérités $(\forall \alpha \exists \beta \exists \gamma((\alpha \Rightarrow \beta \& \alpha, \beta \Rightarrow \gamma))$.

75. Bolzano, 1837, $\mathbb{2} 216$; Notons toutefois que Bolzano ne distingue pas clairement entre fondement complet et partiel dans ce passage.

76. La relation de fondation médiate est introduite aux sections $\mathbb{S} 216$ et 217 de la Wissenschaftslehre. À certains endroits des sections $\mathbb{S}$ 216-19, Bolzano semble suggérer que la relation de fondation médiate à laquelle il pense survient entre des collections de vérités d'un côté et une vérité unique de l'autre. Mais il ne peut réellement vouloir dire cela, puisqu'une telle relation ne serait pas transitive (et nous verrons bientôt que Bolzano admet clairement la transitivité). 
médiat de $\beta$ » ou « $\beta$ est médiatement fondé par $\alpha$ ». On peut, sur cette base, analyser l'idée de «chaîne » en stipulant que la relation de fondation médiate est la clôture transitive de la fondation immédiate partielle (c'est-à-dire la relation dénotée par $«<»)$. Formellement, nous pouvons formuler cela de la manière suivante :

Axiome pour $« \sqrt{ } »: \alpha \sqrt{ } \beta \leftrightarrow \alpha \neq \beta \& \exists n \exists \gamma_{1}-\gamma_{n}\left[\gamma_{1}=\alpha \& \gamma_{n}=\beta \& \forall i<n\left(\gamma_{i}\right.\right.$ $\left.\left.<\gamma_{i+1}\right)\right]$.

Notez que, à strictement parler, « $\alpha \sqrt{ } \beta$ » est l'abréviation de « $\alpha$ est un fondement médiat complet ou partiel de $\beta{ }^{77}$.

Bolzano nomme la manière dont sont connectées les parties d'une collection de vérités données par la relation de fondation médiate la connexion objective entre ces vérités. Elle peut être représentée par une structure arborescente comme suit :

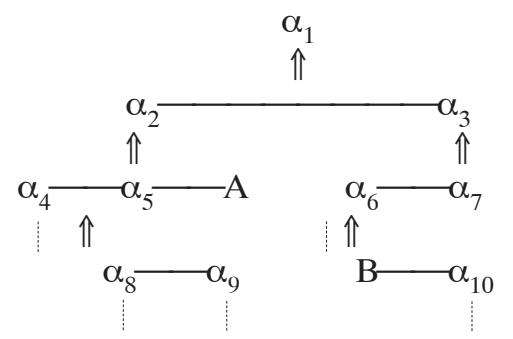

Ici, la relation de fondation immédiate est représentée par « ». Les lignes horizontales indiquent que les (collections de) vérités connectées entre elles fondent ensemble - en tant que collection - une autre collection de vérités. Les points indiquent que la collection de vérités pertinentes sont, elles aussi, fondées. Les vérités individuelles A et B sont des vérités primitives, c'est-à-dire qu'elles n'ont pas d'autre fondement. (Dans la partie suivante de l'article, je discuterai les arguments de Bolzano en faveur de l'existence de vérités primitives.)

Sur la base de ce schéma, il est facile de voir qu'une collection de propositions $\alpha$ est un fondement médiat d'une autre collection de propositions $\beta$ si, et seulement si, il y a une "chaîne " qui relie immédiatement les éléments d'une collection de propositions $\alpha$, ou une partie (propre ou impropre) de $\alpha$, à $\beta$. Ainsi, dans l'exemple ci-haut, $\alpha_{9}$ par exemple, fonde médiatement $\alpha_{5}, \alpha_{2}$, et $\alpha_{1}$. $\alpha_{1}$ est également fondée par $\alpha_{2}, \alpha_{4}, \alpha_{5}$ et $\mathrm{A}$, ainsi que par la collection de, disons, $\alpha_{4}$ et $\alpha_{5}$, ou $\alpha_{4}$ et A. Supposons que $\alpha_{9}$ est la collection de

77. Tel que Bolzano le montre lui-même à la section $\mathbb{} 220$ de la Wissenschaftslehre. Notons aussi que Bolzano utilise le mot «dépendance" (Abhängigkeit) pour la relation de fondation médiate. Il dit aussi que les parties de b «supportent » ou "aident » les vérités de a (elles sont des Hülfswahrheiten) (Bolzano, 1837, \$217). Je n'emploie pas cette terminologie car elle ne reflète pas le point de distinction entre la fondation médiate et immédiate (l'idée de "chaîne " déjà mentionnée). 
vérités $C$ et $D$, alors $C$ et $D$ fondent médiatement tout ce qui est médiatement fondé par $\alpha_{9}$ elle-même.

L'axiome de la fondation médiate implique que la fondation immédiate, de même que la relation d'un fondement partiel à sa conséquence complète, sont des cas spéciaux de la fondation médiate, c'est-à-dire qu'il est suffisant pour qu'il y ait fondation médiate qu'il y ait ces relations, et par conséquent :

Théorème $7: \forall \alpha \forall \beta\left(\left(\alpha \Rightarrow \beta \vee \alpha_{<} \Rightarrow \beta\right) \rightarrow \alpha \sqrt{ } \beta\right)$ (inclusion).

(Si $\alpha \Rightarrow \beta$ ou $\alpha_{<} \Rightarrow \beta$, alors $\mathrm{n}=2$ rend vraie la seconde partie de l'axiome.) Cette conséquence s'accorde avec ce que dit Bolzano. Au début de la section \$217 de la Wissenschaftslehre, par exemple, il introduit explicitement un terme pour toutes les (collections de) vérités qui sont impliquées dans ce qu'il appelle la "remontée de la conséquence au fondement». Néanmoins, cela peut être considéré comme un défaut terminologique. L'usage naturel de «médiat» et «immédiat » suggère qu'être un simple fondement médiat exclue la possibilité d'être un fondement immédiat. Malheureusement, je ne connais aucune alternative terminologique qui n'ait aucun inconvénient.

De l'axiome de la fondation médiate, il découle manifestement que la relation est irréflexive ${ }^{78}$ :

Théorème $8: \forall \alpha \neg(\alpha \sqrt{\alpha})$ (irréflexivité).

Cela implique aussi manifestement que le fondement médiat est transitif, donc

Théorème 9: $\forall \alpha \forall \beta \forall \gamma((\alpha \sqrt{\beta} \& \beta \sqrt{ } \gamma) \rightarrow \alpha \sqrt{ } \gamma)$ (transitivité).

Sur la base de ce qui précède, on peut aisément prouver

Théorème $10: \forall \alpha \forall \beta((\alpha \sqrt{ } \beta \rightarrow \neg \beta \sqrt{ } \alpha)$ (asymétrie).

Supposons que la fondation médiate n'est pas asymétrique. Il y aurait alors des collections de vérités $A$ et $B$ telles que $A \sqrt{ } B \& B \sqrt{ } A$. Mais cela implique, en vertu du théorème 9 , que $A \sqrt{ } A$, ce qui contredit le théorème 8 .

Dans la section précédente, j'ai mentionné que le concept de fondation immédiate, parce qu'il est intransitif, ne semble pas être la façon appropriée d'interpréter "parce que" dans des énoncés qui reflètent des causes et effets. Puisque la fondation médiate, par contraste avec la fondation immédiate, est transitive, elle nous fournit les moyens d'interpréter ces énoncés d'une façon qui s'accorde avec notre usage ordinaire de ces concepts. De plus, les exemples énumérés à la section 3 montrent, selon moi, que nous n'utilisons pas toujours «parce que» dans le sens strict de la fondation immédiate.

Il y a une autre raison de croire que, dans le langage ordinaire, "parce que» exprime le concept de fondation médiate, et non celui de fondation immédiate : nous ne savons habituellement pas si les deux collections de vérités sont reliées en tant que fondement complet et conséquence complète. Même un philosophe aguerri aura de la difficulté à trouver quelques exemples

78. Cf. Bolzano, 1837, $\mathbb{} 218$. 
moindrement plausibles. Si «parce que» exprime le concept de fondation immédiate, il sera difficile d'identifier un minimum de cas où nous avons effectivement raison de faire une affirmation de la forme "parce que». Le concept de fondation médiate, au contraire, n'a pas de critères d'application aussi élevés. Il suffit pour l'appliquer qu'une collection de vérités soit un fondement médiat partiel d'une autre.

J'en conclus par conséquent que si l'hypothèse de base de Bolzano, à savoir que "parce que» dans son usage habituel dans le langage ordinaire met en jeu une relation entre des vérités, doit être vraie alors le candidat le plus plausible au rôle de signification de ce concept est celui de fondation immédiate. Cependant, il est clair que le travail de pionnier de Bolzano ne fournit pas une théorie qui soit complète et satisfaisante. Bolzano en était d'ailleurs lui-même parfaitement conscient. Dans l'introduction qui précèdent sa discussion de la fondation dans la Wissenschaftslehre, il écrit :

Puisque les investigations qui m'occuperont maintenant sont très difficiles et qu'il n'y a quasiment pas de travaux préalables sur ce sujet que j'eusse pu utiliser, je prie le lecteur de ne chercher dans cette section, et ce encore moins que dans les précédentes, quelque achèvement. Presque tout ce que j’ai à dire est à mes propres yeux tissé d'incertitudes; il y a des choses sur lesquelles de n'ose poser aucun jugement définitif et, dans le meilleur des cas, mes investigations ne sont que fragments et ébauches qui auront servi leur but si elles fournissent à d'autres un motif pour réfléchir plus amplement sur ces objets ${ }^{79}$.

(Traduit de l'anglais par Mélissa Thériault)

\section{Bibliographie}

Aristotele, Métaphysique, trad. fr. Jean Tricot, paris, Vrin, 1970.

Armstrong, David, A World of States of Affairs, Cambridge, Cambridge University Press, 1997.

Behboud, Ali, "Remarks on Bolzano’s Collections ", Grazer Philosophische Studien, vol. 53, 1997, p. 109-116.

Bolzano, Bernard, Bernard Bolzano-Gesamtausgabe, dir. E. Winter, J. Berg, F. Kambartel, J. Louzil, B. van Rootselaar, Stuttgart-Bad Cannstatt, 1969-.

Bolzano, Bernard, Wissenschaftslehre, Sulzbach, Seidel, 1837.

79. Ibid., $\$ 195$. Cet article est issu de deux projets de recherche réalisés à l’Université de Genève et dirigés par Kevin Mulligan : le projet Histoire et actualité de la philosophie autrichienne (financé par le Fonds National Suisse de la Recherche Scientifique, subvention numéro 11-55845.98), et le projet IRIS sur les concepts formels. Fabrice Correia, Philipp Keller, Götz Klages, Wolfgang Künne, Dan Lopèz de Sa, Kevin Mulligan, Maria van der Schaar, Benjamin Schnieder, Mark Siebel, Göran Sundholm, et Mark Textor m'ont été d'une grande aide en ce qui concerne le sujet de cet article, et je les en remercie. J'aimerais également remercier les membres du groupe de discussion de Hans-Ulrich Hoche et d'Ulrich Paredy à la Ruhr-Universität Bochum pour une discussion animée de cet article. 
Bolzano, Bernard., Athanasia oder Gründe für die Unsterblichkeit der Seele, Sulzbach, Seidel,1838.

Bolzano, Bernard, "Einleitung zur Größenlehre », dans Bernard BolzanoGesamtausgabe vol. 2A 7.

Bolzano, Bernard, Paradoxien des Unendlichen, Leipzig, 1851.

Bolzano, Bernard, Lehrbuch der Religionswissenschaft, Sulzbach, Seidel, 1834.

Bolzano, Bernard, Bolzano's Wissenschaftslehre und Religionswissenschaft in einer beurtheilenden Uebersicht, Sulzbach, Seidel 1841.

Bolzano, Bernard, "Versuch einer objectiven Begründung von der Lehre von der Zusammensetzung der Kräfte", dans Bernard BolzanoGesamtausgabe, vol. 1/18.

Buhl, Gunter, Ableitbarkeit und Abfolge in der Wissenschaftstheorie Bolzanos, Köln, 1961 (=Kantstudien, suppl. vol. 83).

Correia, Fabrice, "Dépendance existentielle, fondation et objets composés », (à paraître) dans Travaux de logique.

Gentzen, Gehrard, "Investigations into Logical Deduction» (1934), dans The Collected Papers of Gerhard Gentzen, éd. par M.E. Szabo, Amsterdam, North-Holland, 1969.

Krickel, Franck, Teil und Inbegriff, St. Augustin, Academia, 1995.

Künne, Wolfgang, "Propositions in Bolzano and Frege ", Grazer Philosophische Studien 53, 1997, p. 203-240.

Künne, Wolfgang, Conceptions of Truth, Oxford, Oxford University Press, (à paraître).

Mulligan, Kevin; Simons, Peter et Smith, Barry, «Truth-Makers », Philosophy and Phenomenological Research 44 (1984), p. 278-321.

Siebel, Mark, Der Begriff der Ableitbarkeit bei Bolzano, Sankt Augustin, Academia, 1996.

Simons, Peter, Parts, Oxford, Oxford University Press, 1987.

Simons, Peter, "Bolzano on Collections ", Grazer Philosophische Studien 53, 1997, p. 87-108.

Tatzel, Armin, «Proving and Grounding. Bolzano's Theory of Grounding and Gentzen's Normal Proofs ", (à paraître) dans History and Philosophy of Logic.

Textor, Mark, Bolzanos Propositionalismus, De Gruyter, Berlin/New York, 1996. 